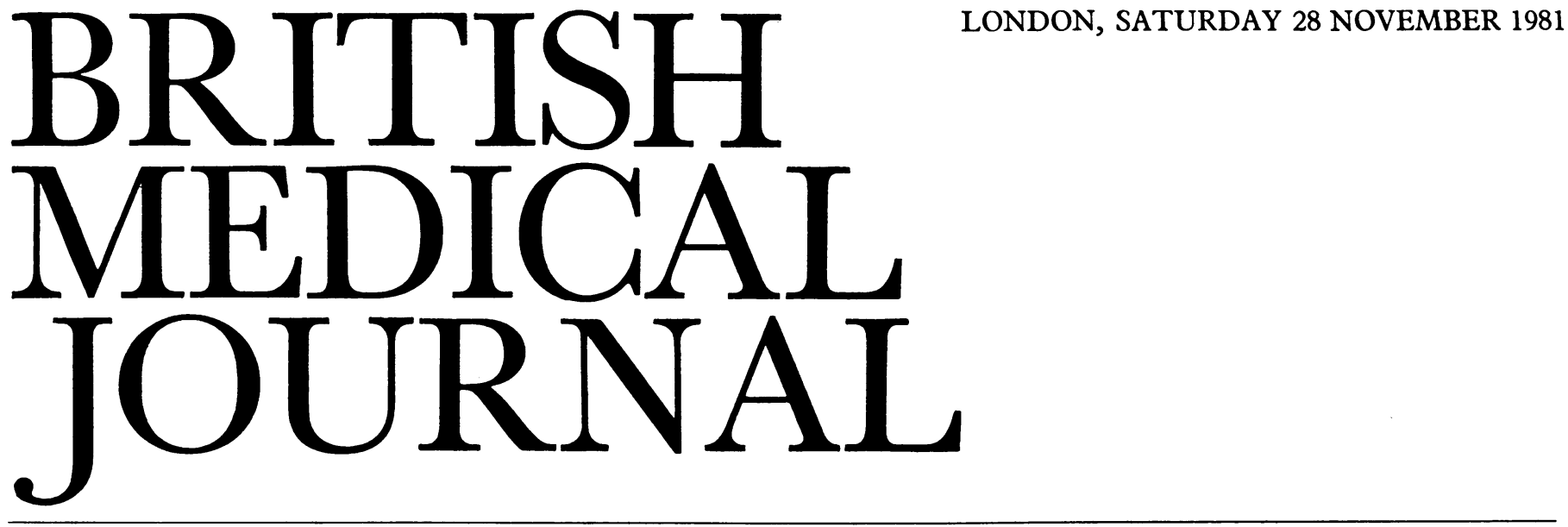

\title{
Avoidable cancer risks with special reference to occupational factors
}

The debate in the United States concerning the likely contribution of occupational factors to the incidence of cancer and its mortality has been furious and often vitriolic. In the wake of this Sir Richard Doll and Richard Peto were commissioned by the Office of Technology Assessment of the United States Congress to provide background material relevant to the assessment of environmental cancer risks of all kinds. They tackled the monumental task by first dividing environmental factors into 12 different categories and then, in the light of the evidence, estimating the percentage of current cancer mortality in the United States caused by each category. In reaching their estimates they attach far more weight to epidemiological data than to evidence from animal and other laboratory studies, since, they argue, these "cannot provide reliable risk assessments." Their best estimates of the contributions to cancer deaths by the 12 categories of factors, as summarised in a single table at the end of their recently published report, ${ }^{12}$ have important implications not only in the United States and not only in relation to occupation.

Not unexpectedly, tobacco, with an estimated contribution of $30^{\circ} \%$ of all cancer deaths, comes out as the single most important identifiable factor. In contrast, occupation is estimated as contributing only $4 \%$ (with a range of "acceptable estimates" of $\left.2-8^{\circ}{ }_{1}\right)$. The estimated contributions of other factors come out in almost the opposite order to that which certain pressure groups and the media commonly present to the layman. Thus pollution accounts for $2 \%$ of cancer deaths, medicines and medical procedures $1 \%$, and industrial products and food additives each less than $1 \%$. In the case of food additives their use, particularly that of antioxidants and preservatives, might even, the report suggests, be protective. Ionising radiation and exposure to ultraviolet light between them were considered responsible for $3 \%$ of cancer deaths (though most skin cancers induced by sunlight are not fatal). Different patterns of sexual behaviour (for example, age at first intercourse and number of sexual partners) and reproductive behaviour (age at first pregnancy) are associated with sizable differences in risk of cancers of the uterine cervix, uterine body, and breast. The estimate of a $7 \%$ contribution to overall cancer risks from such factors is rather plucked from the air, as is a guess of $10 \%$ for infections, including virus infections, but there is a more solid basis for the estimate of a $3 \%$ contribution from alcohol. The risk of cancers of the mouth, pharynx, larynx, oesophagus, but not of the liver, associated with alcohol is much higher in smokers than in non-smokers so that mortality from these causes might be reduced by avoiding smoking even if alcohol consumption is unchanged. Doll and Peto think that alcohol itself rather than other chemicals present in alcoholic beverages is the risk factor; but they suggest that it probably acts by an epigenetic mechanism-for example, by facilitating contact between carcinogens in tobacco smoke and stem cells in the lining of the upper digestive tract.

Unquestionably, it is the estimate of a $35 \%$ contribution to overall cancer mortality from dietary factors which presents the greatest challenge to existing patterns of governmental regulatory activity and allocation of research resources. Epidemiological research on dietary factors is made both difficult and frustrating by the wide variation in what people eat, how much they eat, and patterns of meals, combined with their poor recall of what they ate in the past and the difficulty of checking the accuracy of statements even about current eating habits. For this reason, Doll and Peto think that their estimate of $35 \%$ may be too high by a factor of 3.5 or too low by a factor of 2 . Possible mechanisms include contamination of raw foodstuffs with carcinogens, production of carcinogens during cooking, endogenous production of carcinogens from foodstuffs by bacterial enzymes in the gut, stimulation of metabolic activation, blocking of detoxification, lack of protection afforded by adequate vitamin $\mathrm{A}$ intake, and overnutrition.

In laboratory animals simple overfeeding leading to obesity is strikingly associated with endocrine disturbances and increased risk of a wide variety of neoplasms. Because of the difficulties of measuring how much people eat the best one can do in man is to look for associations between cancer risk and obesity. In the large American Cancer Society study ${ }^{3}$ positive correlations were found between excessive body weight and cancers of the endometrium, gall bladder, uterine cervix, colon and rectum, breast, ovary, and other sites. Armstrong ${ }^{4}$ reported that after the menopause the concentration of circulating endogenous oestrogen is directly proportional to the degree of adiposity. This and evidence from animal studies ${ }^{5}$ suggest that the enhancement of cancer risk associated with overnutrition may be mediated, in part at least, by changes in hormonal state. Apart from this, cross- 
cultural comparisons have consistently shown strong correlations between fat consumption per head and mortality from colorectal, breast, and endometrial cancers.

The important question is how much reliance can and should be attached to the "guesstimates" of Doll and Peto? Plainly, a lot more information is needed before the true contribution of dietary factors can be assessed with any real confidence. Nevertheless, for tobacco, alcohol, and occupational factors, their estimates based on substantial data command considerable confidence, and their 35\% estimate for dietary effects is, in fact, less than the $50 \%$ estimate reached by the American Health Foundation at its 1979 conference. ${ }^{6}$ The fact that no fewer than 11000 of the 17000 annual deaths from cancer in the United States which Doll and Peto attribute to occupation are of the lung emphasises the importance of trying to quantify the relative contributions of smoking and of specific industrial factors, since in some cases the two seem to act synergistically. Thus one of the main recommendations made by Doll and Peto is that a nationally representative case-control study of lung cancer in relation to smoking and occupation should be carried out in the United States.

The fear has often been voiced that the incidence of cancers of all kinds, or cancers of particular kinds, is increasing. Except for cancers of the lung this fear is not substantiated by the data for the United States. Indeed, for some sites-for example, the stomach-the opposite is certainly true. The reliability of long-span time trend data is seriously limited by factors such as changing diagnostic criteria and increasing efficacy of treatment. This problem can partly be overcome by looking only at short-span time trends and comparing patterns in older and younger people. For virtually all types of cancer the age-standardised risk of death increases with age in any one cohort defined by date of birth. The effects of a newly introduced cancer hazard are therefore first seen as increasing death rates in younger subjects-for example, under 50 years of age-in successive cohorts. Similarly, this is where the benefits of a reduction in cancer hazard are most likely to be first seen. It is thus very encouraging that Doll and Peto found downward trends in the United States data, which they consider cannot be explained by improved treatment, for mortality from cancers of most sites in men and women aged under 65. In Britain, downward trends in the mortality from lung cancer are evident for men up to the age of 70 and for women up to the age of 50. In the United States a similar downward trend for lung cancer is now evident for men up to the age of 50 but is scarcely yet discernible in women. The differences between the two countries are readily explained by differences in the past pattern of cigarette consumption and the downward trends in both countries by the reducing tar deliveries of manufactured cigarettes.

But there is nothing in Doll and Peto's review that savours of complacency. Even a $2 \%$ contribution of occupational factors to cancer risk means 8000 cancer deaths a year in the United States and in some cases very high risks among small groups of workers. Research and regulation to eliminate this contribution to the overall cancer death toll therefore merit priority. Also, the authors recognise that in trying to cover the waterfront they are bound to have offended specialists in many disciplines by a somewhat swashbuckling approach to the handling of complex data and theories. They apologise for ranging so widely, citing Bertrand Russell, who also acknowledged that he had done the same. ${ }^{7}$ Be that as it may, most interested specialists will recognise that this attempt to deal with the whole of a vastly complex subject in little over
100 pages is as objective as it is bold. The authors' conclusions are based partly on data summarised in numerous tables and text figures and the use of standard statistical and epidemiological methods and partly on a wide knowledge and understanding of recent published findings in cancer research. Their main achievements are to provide both a structure which can be built on or changed as new knowledge becomes available and a clear strategic view of what is presently known and not known about environmentally determined cancer.

Francis J C RoE

Adviser in Experimental Pathology and Cancer Research, 19 Marryat Road, London SW 19 5BB

${ }^{1}$ Doll $\mathrm{R}$, Peto R. The causes of cancer: quantitative estimates of avoidable risks of cancer in the United States today. Fournal of the National Cancer Institute 1981;66:1191-308.

2 Doll R, Peto R. The causes of cancer. London: Oxford University Press. (In press.)

${ }^{3}$ Lew EA, Garfinkel L. Variations in mortality by weight among 750000 men and women. $\mathcal{f}$ Chronic Dis $1979 ; 32: 863-6$.

- Armstrong BK. The role of diet in human carcinogenesis with special reference to endometrial cancer. In: Hiatt $\mathrm{HH}$, Watson JD, Winsten JA, eds. Origins of human cancer. New York: Cold Spring Harbor Laboratory, 1977:557-65.

${ }^{5}$ Roe FJC. Are nutritionists worried by the epidemic of tumours in laboratory animals ? Proc Nutr Soc $1981 ; 40: 57-65$.

${ }^{6}$ American Health Foundation. Conference on primary prevention of cancer: assessment of risk factors and future direction, June 1979. Prev Med 1980;9:165-332.

${ }^{7}$ Russell B. History of Western philosophy. London: George Allen and Unwin, 1946.

\section{Old skin}

No one dies of old skin. So runs an aphorism now maturing into a cliché, much used at conferences on aging. It also fits the general rule that the harmful effects of aging occur mainly in cells that have been programmed during development to cease mitosis. Human epidermal cells continue to divide right into extreme old age, though the process does slow down, as judged by the low proportion of cells in elderly skin that become labelled after injecting tritiated thymidine intradermally, ${ }^{1}$ and by the slow elimination of a fluorescent dye, dansyl chloride, which binds specifically to the stratum corneum. $^{2}$ Nevertheless, the appearances under light and electron microscopy of the epidermis of an old person remain closely similar to those of young skin, ${ }^{3}$ provided that the comparison is made on specimens from areas normally covered by clothing (so avoiding any muddle with changes due to prolonged exposure to the sun). The viable epidermis appears a little thinned, but the stratum corneum does not, and its cardinal function, the ability to restrict loss of water, is not affected. ${ }^{1}$ It is only by refined techniques that the number of cells in the epidermis and their height can be shown to diminish a little with age, though oddly the surface area of individual cells in the stratum corneum actually increases. ${ }^{4}$

More interesting changes appear when the epidermis is split off and examined from below with the scanning electron microscope. In the young skin the fine villous protrusions (microfeet) of the basal cells into the dermis give the undersurface of the epidermis a distinctive velvety appearance; this is almost lost in old age. In addition, the complex mixture of valleys and ridges, easily seen on the underside of young epidermis, becomes progressively flattened in old age. ${ }^{5}$ These changes may explain why suction blisters can be raised so easily in the elderly. 